\title{
Smart Indoor Positioning/Location and Navigation: A Lightweight Approach
}

\author{
José Antonio Puértolas Montañés ${ }^{1}$, Adriana Mendoza Rodríguez ${ }^{2}$, Iván Sanz Prieto ${ }^{3}$ \\ ${ }^{1}$ Bizeu Consulting, ${ }^{2}$ University Complutense of Madrid, ${ }^{3}$ AXA Tech
}

\begin{abstract}
In this paper a new location indoor system is presented, which shows the position and orientation of the user in closed environments, as well as the optimal route to his destination through location tags. This system is called Labelee, and it makes easier the interaction between users and devices through QR code scanning or by NFC tag reading, because this technology is increasingly common in the latest smartphones. With this system, users could locate themselves into an enclosure with less interaction.
\end{abstract}

Keywords - Indoor location system, NFC technology, QR codes.

\section{INTRODUCTION}

I NDOOR Positioning shall be defined as any system which attempts to provide an accurate positioning inside of a covered structure; it is used to locate people or required objects in large buildings and in closed areas/spaces. At present, there are several types of location-sensing systems, each having their own strengths as well as limitations. [1]Indoor location sensing systems have become very popular in recent years, because many applications need to know the physical location of objects. These systems provide a new layer of automation called automatic object location detection[2] and use wireless concepts, optical tracking or ultrasonic techniques.[3]

The relevance of context-awareness for mobile users has been demonstrated in several applications which have been summarized in applications of context-aware computing, and have included fieldwork, museums, route planning, libraries and tourism.[4] There are three technologies commonly used for indoor location systems -ultrasonic, infrared and radio frequency. These can be supplemented by inertial systems which are generally used for prediction. [5]

One of the most well-known location-based systems is the Global Positioning System (GPS), which is widely used to track moving objects located outdoors and determines the position (altitude, longitude and latitude) of an object in any point of the planet by triangulation [6]. However, GPS, as it is satellite dependent, has an inherent problem of accuracy, when determining the location of objects inside buildings. Active

${ }^{1}$ Line of sight is a type of propagation that can transmit and receive data only where transmit and receive stations are visible each other without any sort of an obstacle between them.
Bat and Cricket, are cited as [7] use ultrasonic pulse TDOA to enable high precision and dimensional positioning in indoor environments. Although, ultrasonic positioning system requires additional hardware to send and receive ultrasonic pulse, it can determine 3D position of indoor objects with an accuracy of a few $\mathrm{mm}$ to a few $\mathrm{cm}$. [1]

Indoor location technologies may be also classified according to the location positioning algorithm, the physical layer or location sensor infrastructure. Locations sensing approaches can be classified as follows: location fingerprinting (scene analysis), triangulation, trilateration, hyperbolic lateration, proximity, and dead reckoning. [8]

In this paper/article, Indoor positioning systems are classified according to the technology on which they are based. There are systems which use Wireless networks, Satellitebased techniques, infrared systems, ultrasonic systems, Ultra Wide Band (UWB), RSS, hybrid systems, GPS indoor, Bluetooth...

Technical deployment of indoor positioning systems involves significant investment. Also these systems have a lot of problems like multipath effects, they need a specific infrastructure, require a Line Of Sight ${ }^{1}$ (LOS) etc. also they consume battery of mobile devices when obtaining the information of indoor location.

We present an indoor positioning and orientation system, which optimize the user mobility in closed spaces. This technology employs a location codes system. It is a simple and inexpensive solution to obtain the indoor location and orientation.

This paper is organized as follows. Section 2 presents relevant overview of existing indoor location systems, section 3 explains the most common problems of indoor location systems, section 4 describes the indoor position system based in QR codes. Finally, section 5 ends the paper with a discussion about future work and conclusions.

\section{OVERVIEW OF EXISTING INDOOR LOCATION SYSTEMS}

Physical location class can be broken down into three subcategories: describe locations, spatial locations and network locations:

- Location related to geographic objects such as mountains, lakes, cities, roads, countries or other structures that have a description such as name, identifier, or number is known as a descriptive location. 
- Spatial location is used more in professional applications where a descriptive location does not provide enough details.

- Network location refers to a location based on the topology of a communications network. A user's device position in a network is achieved based on its Internet Protocol (IP) address. However in mobile networks a network location is achieved from the base stations used by the mobile terminal. [8]

The techniques used to estimate location in a cellular network have been successfully used in wireless Local Area Networks (WLANs), Bluetooth, Ultra-wideband (UWB), Ultrasound, Radio Frequency Identification (RFID), Hybrid technologies, and QR scanning method.

\section{- Systems based on WLANs:}

In the last few years, WLAN radio-signal-based positioning systems, supported by underlying Radio Frequency (RF) and Infra-Red (IR) transmission technologies has experimented an enormous expansion and is expected to continue this trend due to the fact that it is an economical solution providing convenient connectivity and high speed links, and can be implemented with relative ease in software.

Wi-Fi RTLS (Real Time Location Systems) enables position sensing by using existing wireless LAN routers [9]. Additionally, WLAN covers a large area and is not restricted by line of sight issues. A WLAN can support a large number of nodes and vast physical areas by adding access points to extend coverage. Therefore, WLAN allows users to be truly mobile as long as the mobile terminal is under the network coverage area. [4]

WLAN is appealing because it allows enhanced connectivity and is particularly useful when mobile access to data is necessary. Additionally, user flexibility and probability can easily be reconfigured while requiring no cable infrastructure.[4]

The Wave LAN system triangulates 2D position of an object within a building by using either empirical data or a mathematical model of indoor radio propagation. Advantages of this system are such that it requires few base stations and it uses the same general wireless networking in the buildings. [3]

A special case of this technology is a systems based on fingerprinting. Location fingerprinting refers to techniques that match the fingerprint of some characteristic of the signal that is location dependent. The fingerprints of different locations are stored in a database and matched to measured fingerprints of different locations are stored in a database and matched to measured fingerprints at the current location of an MS (Mobile Station). [10]

The fingerprinting technique is relatively simple to deploy compared to the other technique such as angle-of-arrival (AOA) and time-of-arrival (TOA). Moreover, there is no specialized hardware required at the mobile station (MS). Any existing wireless LAN infrastructure can be reused for this positioning system. The most common algorithm to estimate the location computes the Euclidean distance between the measured RSS vector and each fingerprint and database. The coordinates associated with the fingerprint that provides the smallest Euclidean distance is returned as the estimate of the position. [11]

\section{- Systems based on Bluetooth:}

The Bluetooth wireless technology is a technology originally designed as a short-range connectivity solution for personal, portable, and handheld electronic devices.

Bluetooth is lighter standard than WLAN, highly ubiquitous (embedded in most phones, personal digital assistants (PDAs), etc.) and supports several other networking services in addition to IP. Bluetooth tags are small size transceivers. As any other Bluetooth device, each tag has a unique ID. This ID can be used for locating the Bluetooth tag. [2]

The position estimation occurs in the mobile terminal without the need of changes in the already fixed installed network topology. The developed system is based on the known and already published triangulation methods using the received signal power strength of the surrounding Bluetooth access points. These points must exist previously. For precise position estimation, the dependence between the distance and the received signal strength has to be determined. [12]

\section{- Systems based on UWB:}

Ultra Wide band (UWB) impulse radio signals are employed for location and tracking. This system platform was constructed using standard electronics components, therefore, it allows fast performance evaluation and to estimate the time of arrival (TOA) of received pulse signals. Systems based on UWB use receivers deployed in a building to track users carrying small tags which emit ultra-wideband signals. Finally, these signals are transferred to a server where the location of the transmitter is calculated [12].

The main advantage of the UWB signals is to have high temporal resolution and to provide accurate TOA measurements in multi path environments. The transmitted signal has a sequence of short pulses. These are propagated in the media and received by at least four receivers placed in known positions [3].

\section{- Systems based on Ultrasounds:}

Indoor ultrasonic location sensing systems frequently use a number of ceiling-mounted transmitters to measure the position of a user in a room, and they process data from time of flight between transmitters and this user. Usually, these systems are composed by a hardware, consisting of transmitters or beacons which emit signals; software-based algorithms of location and at least one receiver that the user must carry. These receivers can take several forms depending on the system used.

For example, the Cricket system has an accuracy of $1 \mathrm{~m}$ to 3 $\mathrm{m}$ and supports multiple target discoveries. The user's position is not shared unless the user accepts advertising that 
information. Thus there are no privacy issues due to the location being estimated on the mobile device.

Cricket's limitations are the absence of a centralised management and monitoring functions. The computational requests are also quite high, which means high power consumption in order to synchronise the ultrasound pulse an RF data.

Another system based on Ultrasounds is called Bat system. This system consist that users wear small badges which emit an ultrasonic pulse when are radio-triggered by a central controller. The system determines the time of flight of the pulses from the badges to a network of receivers on the ceiling, and calculates the 3D positions of the badges using a multilateration algorithm. [13]

\section{- Systems based on RFID:}

Radio Frequency Identification (RFID) is a rapidly developing technology for automatic identification of objects [12]. The RFID position estimation is based on electromagnetic communication between RFID readers and tags, so RFID readers can read data emitted from RFID tags. Also, it is becoming more common that mobile devices have Near Field Communication (NFC) reader based on RFID technology, which allows reading these tags [13].

NFC technology is a bidirectional short range, wireless communication technology. Therefore, the communication occurs between two nearby devices: on one hand, a mobile phone acting as a NFC reader, and on the other hand, RFID tags, which are processed by the device. Systems based on NFC technology orient the user by gathering his destination point; this way the mobile device collects the current position from NFC tags, so the system computes the optimal route. Finally, another factor to keep in mind is that this technology can operate in card emulation, reader / writer, and peer-to-peer (P2P) modes [14].

RFID tags are categorized as either passive or active. Active tag contains embedded batteries in order to provide a much broader scanning range. The major disadvantage of using active RFID tag based solutions is the high cost of the active tags. [14]. In contrast, passive tags operate without a battery and are mainly used to replace the traditional barcode technology. They are much lighter and less expensive than active tags, offering a virtually unlimited operation lifetime. However, their read ranges are limited, offering a less range than active tags [7]. The employed signal technologies include RF (radio frequency) such as ultrasound, infrared, vision-based systems and magnetic fields[15].

The main advantages of this technology, is that non-contact is required and not need direct vision (N-LOS) [3]. Therefore, RFID systems have been widely adopted as an attractive technology for many significant applications such as asset tracking and industrial automation [13].

\section{- Systems based on hybrid technologies:}

Hybrid systems combine two or more technologies in order to improve the accuracy and precision of the location estimation. Depending on used technologies by these systems, they will have certain advantages or disadvantages. These technologies have been described in a bellow section.

An example of hybrid systems is the system called indoor GPS. In this system, user position tracking is mainly defined by at least four (or more) transmitters and a receiver. A battery operated transmitter uses laser and infrared light to transmit one-way position information and elevation from the transmitter to the receiver. The receiver has photodiodes inside its module and senses the transmitted laser and infrared light signals. The signal is transferred through a wireless network connection providing mobility to users. The $3 \mathrm{D}$ position of the optical receiver is then calculated by the process of triangulation. Triangulation is used if the angles to known ( $\alpha$ and $\beta$ ) are given. With two known locations, the absolute position in $2 \mathrm{D}$ can be determinate. The two angles are used to determinate the line-of-sights (LOS) for each of the known locations. Indoor GPS, unlike UWB, depends on a clear line of sight and some calibration points are required. However, both technologies offer centimetre level positioning accuracy with Indoor GPS positioning offering significantly higher precision.[4]

\section{- Systems based on QR scanning method:}

QR-Codes are a type of visual tag, like a 2D barcodes, since they are more efficient than traditional tags and have a much higher information capacity. Due to the open QR-Codes specification, these codes can be employed without restriction and for all applications. Many mobile services rely on them; indeed it has been proposed that they should be embedded in images, such as those used on printed paper tickets. [16]

QR-Maps, is a solution to obtain indoor user locations using QR-Codes (Quick Response Codes) and Google Maps API. Since a smartphone is available, a QR-Code with a short text can be decoded. This text is sent to a location server, which returns an URL showing the map centred in the specific location[16]. Thus, no special hardware is required, simply a smartphone with a camera capture QR-Codes and Internet connection to access the map.[17]

There is another system which combines all previously mentioned features (QR scanning, do not required any specific API installed; do not need any transmitters and receiver, etc.). This system is called Labelee and it based on scanning QR codes, which allows the location and position in indoor environments.

The main improvement regarding the system described above is that Labelee allows showing the position at indoor environments, therefore users knows their position and it will be much easier to know where they would like to go.

Indoor position systems could be classified in active or passive systems. Passive location represents the estimation of a person's position without require of a physical device, for example, a tag or sensor. However, most of location techniques are part of the active systems class, due the need of people who want to locate themselves into an enclosure to carry an electronic device or another object which allows 
estimating their position.[8] Table 1, summarizes the main characteristics of the technologies described above:

\section{TABLLE 1: VARIABLES OF THE TECHNOLOGIES USED ON INDOOR POSITIONING SYSTEMS}

\begin{tabular}{|c|c|c|c|c|c|c|}
\cline { 5 - 7 } & \multicolumn{2}{c|}{} & \multicolumn{2}{c|}{ Labelee } \\
\hline Outdoor location & WiFi & Bluetooth & UWB & Hybrid S./I. GPS & RFID/ NFC & Scanning/QR \\
\hline Indoor location & $\checkmark$ & $\mathbf{X}$ & $\mathbf{X}$ & $\mathbf{X}$ & $\checkmark$ & $\checkmark$ \\
\hline $\begin{array}{c}\text { Location and Orientation in } \\
\text { Indoors }\end{array}$ & $\mathbf{X}$ & $\mathbf{X}$ & $\mathbf{X}$ & $\checkmark$ & $\checkmark$ & $\checkmark$ \\
\hline Guided indoors & $\checkmark$ & $\checkmark$ & $\checkmark$ & $\checkmark$ & $\checkmark$ & $\checkmark$ \\
\hline Accuracy & $3 \mathrm{~m}$ & $2 \mathrm{~m}$ & $10 \mathrm{~cm}$ & $1 / 5 \mathrm{~m}$ & $0 \mathrm{~m}$ & $0 \mathrm{~m}$ \\
\hline Range & Room Scale & $10 \mathrm{~m}$ & $15 \mathrm{~m}$ & Gobal & $\begin{array}{c}\text { Scale according } \\
\text { enclosure }\end{array}$ & $\begin{array}{c}\text { Scale according } \\
\text { enclosure }\end{array}$ \\
\hline Signal & $\mathrm{RF}$ & $\mathrm{RF}$ & $\mathrm{RF}$ & $\mathrm{RF}$ & $\mathrm{RF}$ & \\
\hline $\begin{array}{c}\text { Necessary Infrastructure } \\
\text { WLAN router }\end{array}$ & Specific Trans & specific Trans. & Specific Trans. & NFC Tag & QR Tag \\
\hline $\begin{array}{c}\text { Battery consumption/Energy } \\
\text { Spending }\end{array}$ & Medium & Medium & Medium & High & \multirow{2}{*}{ Low } & Low \\
\hline Specific Application & $\checkmark$ & $\mathbf{X}$ & $\mathbf{X}$ & $\checkmark$ & $\mathbf{X}$ & $\mathbf{X}$ \\
\hline Cost & High & High & High & High & Low & Low \\
\hline
\end{tabular}

III. REVIEW OF THE MOST COMMON PROBLEMS

Since GPS is not available indoor location, many approaches have been proposed to facilitate indoor location based on wireless technologies such as RFID (radio frequency identification), Bluetooth or IEEE 802.11. All these solutions require the deployment of a wireless infrastructure and, in some cases, specific technologies seldom found in smartphones.[16]

The GPS signal [7] is not usually available in indoor environments. [18] Satellite-based navigation systems require an unblocked line of sight between the satellite and the receiver to function properly. If this prerequisite is not met, satellite navigation is degraded; different methods and technologies are needed.

One way for locating a mobile device in two dimensions requires the measurement of the Line Of Sight (LOS) distance between the mobile phone and at least three participating BSs (Base Stations). Each distance measurement generates an area which is focused at the measuring base station and has a radius equal to the mobile-to-base distance. So, the three regions unambiguously determine the location of the device.

However this may become a problem if the LOS is not continuous. The usage of signals that can penetrate building materials may overcome this problem in the near future[15]. A mobile phone positioning system is accurate to around $100 \mathrm{~m}$. at indoor, short-range (approximately $10 \mathrm{~m}$ radius) wireless cell may be used to determine positions based on cell identification, but requires many stations. [18]

When the range measurements are corrupted by noise, then the location of the mobile station can be determined by finding the solution that is most consistent with the measurements. However, in dense urban environments, there may be no direct path from the mobile to the BS. Due to reflection and diffraction, the propagating wave may actually travel excess path lengths on the order of hundreds of meters and the direct path is blocked. In order to mitigate the effect of the measurement BS, it is necessary to develop location algorithms that are robust to the NLOS (Non Line Of Sight) error.[19]
Another factor that affects the indoor positioning systems is called multipath. This is the propagation phenomenon that results in radio signals reaching the receiving antenna by two or more paths. The multipath transmission (multi path fading) includes large scale fading and small scale fading. Large scale fading corresponds to average signal power attenuation, small scale fading is due to dramatic alterations in amplitude and phase. Almost all indoor position systems have these effects of multipath systems that include constructive and destructive interference, and phase shifting of the signal. Destructive interference cases fading[20]. It is important to note that, RSS measurements are affected by multipath effects or attenuation when passing through walls, distorting them from the expected free space values. [21]

A solution to compensate the multipath effects on received signal, is equalized these effects by using Equal Gain Combining Algorithm further correlating the received signal with reference signal so that we can eliminate other unwanted signals. The problem is that obtained signal has more noise power, so signal may not sufficient to locate Indoor positioning in centimetre level or millimetre level.[20]

Moreover, the cost of a positioning system may depend on many factors. These factors include money, time, space, weight, and energy. The time factor is due to installation and maintenance, also, mobile units may be having tight space and weight constraints. Sometimes, installation of indoor positioning systems may not have to result in additional costs, since previously there could exists hardware being used for another purposes. Energy is another important cost factor. For example, some mobile units like RFID tags are completely energy passive since they only respond to external fields and, thus, could have an unlimited lifetime, unlike most devices with rechargeable battery, which have a lifetime of several hours without recharging [2].

Another factor to consider is complexity of a positioning system. This can be attributed to hardware, software and operation factors. Most of the mobile units lack strong processing power and long battery life; so, positioning algorithms with lower complexity would be preferred. Usually, it is difficult to derive the analytic complexity formula of different positioning techniques; thus, the computing time is considered. Another factor to keep in mind is location rate, which is an important indicator for complexity. The dual of location rate is location lag, which is the delay between a mobile target moving to a new location and reporting the new location of that target by the system.[2]

Apart from the general problems mentioned above, each indoor positioning system, according to the technology on which they are based, has its specific problems, which will be analysed below.

\section{- Inconvenient of WiFi based systems:}

In order to enable the user to locate himself in enclosures, it is necessary that the mobile phone and the network are synchronized; therefore, the device should calculate its distance from the access point (AP). 
The only available information is the signal strength received from each AP. Indeed, the received signal strength is measured. Such information is available because the APs send signals periodically. Mobile devices use those signals to handle the roaming inside the network. Given this consideration, it is possible to get a list of the received power coming from all the APs covering the area where the mobile phone is moving.

The easiest approach for locating a mobile device in a WLAN environment is to approximate its position by the position of the access point received at that position with the strongest signal strength. However the main drawback is its large estimation error. The accuracy is proportional to the density of access points; which is in the range of the 25 to 50 meters for indoor environments. [22]

In order to configure a wireless navigation system in an indoor environment it is required individually encoded transmitters and the setup of a sufficient number of receivers to provide at least room-level resolution. Also, for the navigation environment a map is required, which holds the RSSI at strategic points in that area, usually in form of a grid. The process, during which the RSSI is recorded at each grid point, is called fingerprinting and it is a very time consuming one. It is usually only valid for a particular antenna/access point pair and is very sensitive even to the slightest change in the surroundings (e.g. the removal of a particular item of furniture). Some problems of fingerprinting are that such techniques require specialized hardware in every base station, or access point to correlate the multipath characteristics. In WLANs, an easily available signal characteristic is the received signal strength (RSS) and this has been used for fingerprinting. The RSS is a highly variable parameter and issues related to positioning systems based on RSS fingerprinting are not understood very well. This may not very costly for small areas, but it becomes an increasingly important component as the area to be covered and the number of users becomes large. [10]

We must also take into account a problem with the multi floor environments, because two or more very different locations could potentially have the same RSS locationfingerprint. Instead of storing average RSS values, the joint or marginal distributions of the RSS are used in fingerprinting locations. The experiments are once again conduced in hallways and the accuracy rages between 5 and 20 feet in most cases.

\section{- Inconvenient of Bluetooth based systems:}

This system was not designed taking into account privacy and security topics, due to the installation of Bluetooth beacons that will send advertisements to mobile devices which are within its scope. Also, device address data sent from a Bluetooth Sensor to the Ad Server is not encrypted. [1]

Another disadvantage is the Bluetooth range which depends on the Bluetooth Class. The maximum range is approximately $100 \mathrm{~m}$ for Bluetooth Class 1. The range can be extended with directional antennas and signal amplifiers but this increases the cost to deploy a Bluetooth-based localisation system [7].
Furthermore, the position accuracy is dependent on the amount of cells used [14]

\section{- Inconvenient of $U W B$ based systems:}

Indoor location systems are based on UWB, and have some disadvantages produced by the effects of metallic and liquid materials, these effects can be reduced with a good placement of the UWB readers. UWB technology is suitable for 2D and 3D location estimation. Most common methods used in a UWB localisation system are Uplink-Time Difference of Arrival (TDOA) and Angle of Arrival (AOA). A combination of TDOA an AOA can reduce the number of sensor required for a system using just TDOA.[8]

The problems of using this ultrasonic technique are the requirement of large number of receivers across the ceilings and their placements across the ceiling which needed quite sensitive alignments. [3] These systems are loss of signal due to obstruction; false signals due to reflections; and interference from high frequency sounds. [5]

\section{- Inconvenient of Ultrasound based systems:}

The main issue of using ultrasonic techniques is the requirement of a large number of receivers across the ceiling. Their placement needs some calibration [3]. Other disadvantages is that need a single-user access, if multiple collocated transmitters send at the same time, their signals can interference with one another, and make it difficult for the receiver to distinguish between them. Also, these systems presents a poor performance in the presence on in band noise, this means that, people create ultrasonic noise trough their everyday actions, although they are rarely aware of it because ultrasound is inaudible. [23]

\section{- Inconvenient of RFID based systems:}

A drawback to consider in systems based on RFID technology is the type of label used. Active tags are more accurate, hence their cost is much higher, however, passive tags are more economical, and yet have a lower precision. Also, It must be taken into account the greater the number of labels installed, a larger area will be covered and therefore, the cost will be higher.

\section{- Inconvenient of Hybrid based systems:}

All previously described inconvenient are mixed on hybrid based systems, so it uses these technologies.

\section{- Inconvenient of scanning QR Codes:}

The main disadvantage of methods based on QR codes scanning, is that the determined position is always the position of the marker. So the used navigation device must be placed closed to the marker. The range depends on the resolution of the used camera; the accuracy depends on the accuracy of the measured marker position. [18] 
QR-Codes have been specifically designed for fast information transfer. QR-code data retrieval depends on factors such as camera quality, picture angle or luminosity, as well as on the area of the code. Thus area depends on the size of the data. In our case data size is minimal, just a short text identifying the map and the location, which corresponds to a small QR-Code that can be placed on any wall or displayed on any screen with negligible aesthetic impact.[16]

\section{NEW APROACH TO CREATE A CHEAP, PLATFORM INDEPENDENT AND LIGTH WEIGHT INDOOR POSITIONING SYSTEM}

A recent investigation of Baltimore University shows that our position in known environments is represented in a short of interior map located in cerebral hippocampus, formed by neuronal groups called "places-cells". When we make a walk that we had make before, places cells are activated sequentially in our interior map, showing the right way. [24] Working in a similar way, Labelee employ location tags which contains a QR code, which are distributed at indoor environments, acting as a places-cells, so users only needs to scan the QR code using their devices to find the way to go. These location tags act the same form as location cells, because they encode their position and orientation to allow guidance and navigation.

Labelee is an indoor positioning and orientation system, which optimizes the client/user mobility inside the building. Labelee employs the LC (location codes) system, which includes a QR (quick response) code and a NFC chips that acts as an information point for the user: it stores its geographic position and leads him to its target.

The user only has to take a picture of one of those QR labels or read the NFC chips with his "smartphone", and then the system will place him and lead him in the map, without the need of downloading or installing any app in the mobile phone. Figure 1 shows how Labelee works. Since a user arrives at a closed environment, and wishes to know where he. Users can locate other points of interest in the map.

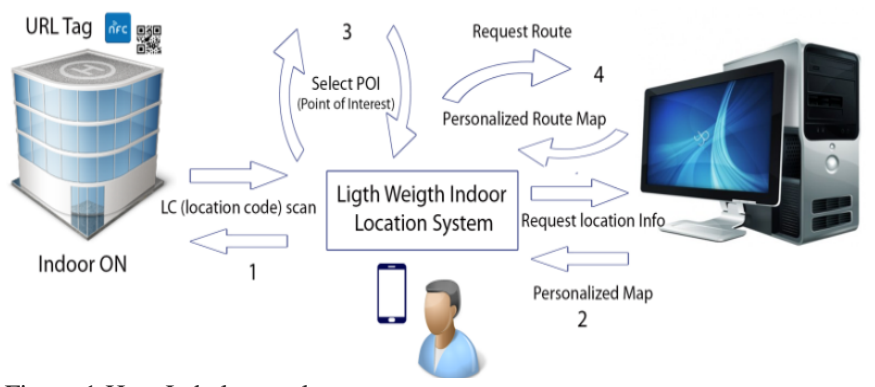

Figure 1:How Labelee works

To know these points, users need to scan the nearest location tag. Once a point of interest is selected, Labelee indicates the current position and the selected point of interest. If the selected point of interest is located on a different floor,
Labelee shows the closer available stairs or elevator and returns a map displaying the shortest way to change floors.

To navigate on indoor environments with Labelee, it is necessary to have all the indoor space information previously processed, in order to calculate all possible routes. Depending on the extent of the environment, or the complexity of these, there will be more or less location tags that correspond to the placement of the labels. Those location labels will be strategically distributed all over the place, when the user scans any location tag, Labelee will indicate his current position and will guide him step by step to his destination. This system works with any smartphone or tablet, even if the smartphone have any camera, just entering the URL which appears besides the QR code. In the figure 2 shows this process.

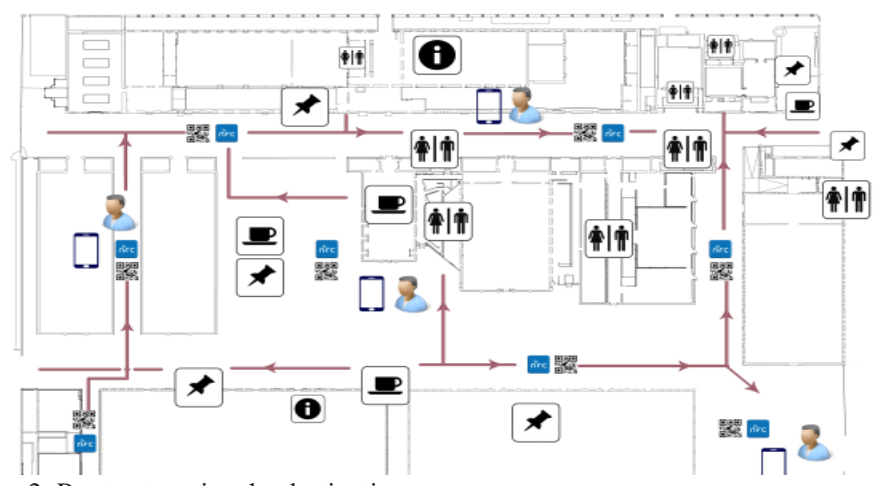

Figure 2: Routes to arrive the destination

Therefore, applications of Labelee are varied and can be installed in any type of area where it needs to have reference plans. This system is very useful, for environments due to the functions they perform, attract different people daily.

In outdoor spaces, Labelee is an alternative in urban historical centres or any place where, due to the morphology or the density of habitants, the GPS signal is not optimal despite the fact is an open space. Also, this solution enables visitors the location, orientation and guidance in public parks, city centres, amusement parks and tourist attractions, apart from showing other routes of various kinds.

Moreover, Labelee is very helpful choice, because it does not rely on any wireless network antennas or transmitters; hence, the required installation is minimal, so it is suitable for shopping centres, parking, airports, museums, hospitals, data processing centres, buildings and office centres, special events (fairs, festival, congresses, conferences, etc.), stadiums, holiday resorts, cruises, etc.

\section{V.CONCLUSIONS AND FUTURE WORK}

This paper has presented an indoor location system based on QR codes and recently RFID technology, through NFC readers, more common in the latest smartphones; users could locate themselves into the environment with less interaction. Moreover, it would be interesting to try to regulate the transmission of location according to the bandwidth, in order to get a greater energy savings and a less data consumption. 
As future work, we intend to use smartphone compasses to show the user how to go the desired destination. With the of help of accelerometers, it will be also possible to estimate walking distances as additional information. Also the management module could be improved to get information from the public which uses different browsers to find the target audience

Some improvements will be shown, for example plant aerial view of buildings, different levels of detail within the map, zoom in the area where the user is situated-so you could get a clearer idea of where is located.

A cartographic base with vector maps is being created at this moment, which will be useful, for example, to remember the position where our car is parked, offer custom routes and maps, different levels of detail inside the map, show distance and time to our destination using 3D maps.

Another important topic is regarding optimize the file sizes, in order to get a better transfer. Also is being done. Also it is being created a test procedure for different mobile platforms, which will serve as the basis for the development of an easyto-use application which will have continuous integration, so eventual mistakes will be corrected very quickly. Incidents resolution and detection will be faster, because events, queries and errors are being defined and stored in a specific database. Location could be also shared, using instant messaging applications, social networks, or just share with tourist information webpages, entertainment, etc. A new web portal with maps will be developed, which will have maps classified by categories from all areas where the system is installed. Therefore users could interact, share or point their places of interest.

Overall, a direct relationship exists between the accuracy and the cost in positioning systems. However, the system described in this article, is considered a fine-grained system, which means that its accuracy can be estimated with a high degree of resolution, with a low cost.

\section{REFERENCES}

[1] [1] L. Escobés, "Summary of the State of the Art in indoor location systems," no. December, pp. 1-3, 2009.

[2] [2] H. Liu, S. Member, H. Darabi, P. Banerjee, and J. Liu, "Survey of Wireless Indoor Positioning Techniques and Systems," vol. 37, no. 6, pp. 1067-1080, 2007.

[3] [3] H. Koyuncu and S. H. Yang, "A Survey of Indoor Positioning and Object Locating Systems," vol. 10, no. 5, pp. 121-128, 2010.

[4] [4] H. M. Khoury and V. R. Kamat, "Evaluation of position tracking technologies for user localization in indoor construction environments," Automation in Construction, vol. 18, no. 4, pp. 444-457, Jul. 2009.

[5] [5] C. Randell, "Low Cost Indoor Positioning System *," no. 15986.

[6] [6] R. G. Crespo, G. G. Fernandez, and O. S. Martínez, "In Premises Positioning - Fuzzy Logic," pp. 284-291, 2009.

[7] [7] Y. Fukuju, M. Minami, H. Morikawa, T. Aoyama, I. Science, and F. Sciences, "DOLPHIN : An Autonomous Indoor Positioning System in Ubiquitous Computing Environment Yasuhiro FUKUJU *, Masateru MINAMI *, Hiroyuki MORIKAWA **, and Tomonori AOYAMA ** Graduate School of Information Science and Technology, The University of Tokyo ** Gr."

[8] [8] G. Deak, K. Curran, and J. Condell, "A survey of active and passive indoor localisation systems," Computer Communications, vol. 35, no. 16, pp. 1939-1954, Sep. 2012.

[9] [9] U. Rueppel and K. Stuebbe, "BIM-Based Indoor-EmergencyNavigation-System for Complex Buildings," Tsinghua Science \& Technology, vol. 13, no. October, pp. 362-367, Oct. 2008.
[10] [10] P. Prasithsangaree, P. Krishnamurthy, P. K. Chrysanthis, T. Program, and I. Section, "ON INDOOR POSITION LOCATION WITH WIRELESS LANS," pp. 720-724, 2002.

[11] [11] K. Kaemarungsi and P. Krishnamurthy, "Modeling of indoor positioning systems based on location fingerprinting," Ieee Infocom 2004, vol. 2, pp. 1012-1022.

[12] [12] S. Feldmann, K. Kyamakya, A. Zapater, and Z. Lue, "An indoor Bluetooth-based positioning system: concept, Implementation and experimental evaluation."

[13] [13] M. Hazas and a. Hopper, "Broadband ultrasonic location systems for improved indoor positioning," IEEE Transactions on Mobile Computing, vol. 5, no. 5, pp. 536-547, May 2006.

[14] [14] B. Ozdenizci, K. Ok, V. Coskun, and M. N. Aydin, "Development of an Indoor Navigation System Using NFC Technology," no. April, pp. 25-27, 2011.

[15] [15] R. Mautz, "Overview of current indoor positioning systems," Geodesy and Cartography, vol. 35, no. 1, pp. 18-22, Jan. 2009.

[16] [16] E. Costa-montenegro, F. J. González-castaño, D. Conde-lagoa, A. B. Barragáns-martínez, P. S. Rodríguez-hernández, and F. Gilcastiñeira, "QR-Maps: an Efficient Tool for Indoor User Location Based on QR-Codes and Google Maps," pp. 928-932, 2011.

[17] [17] Y. Xue, G. Tian, R. Li, and H. Jiang, "artificial object mark in complex indoor environment," pp. 6648-6653, 2010.

[18] [18] C. Lukianto and H. Sternberg, "Overview of Current Indoor Navigation Techniques and Implementation Studies Overview of Current Indoor Navigation Techniques and Implementation Studies," no. May 2011, pp. 18-22.

[19] [19] M. P. Wylie and J. Holtzman, "The non-line of sight problem in mobile location estimation," Proceedings of ICUPC - 5th International Conference on Universal Personal Communications, vol. 2, no. 3, pp. $827-831$.

[20] [20] P. Veeranath, D. N. Rao, S. Vathsal, and N. Bhasker, "Reducing Multipath Effects in Indoor Channel for Analysis of GPS / Pseudolite Signal Acquisition," vol. 3, no. 2, pp. 1-6, 2013.

[21] [21] Y. Á. López, M. E. D. C. Gómez, J. L. Álvarez, and F. L.-H. Andrés, "Evaluation of an RSS-based indoor location system," Sensors and Actuators A: Physical, vol. 167, no. 1, pp. 110-116, May 2011.

[22] [22] F. Evennou and F. Marx, "Advanced Integration of WiFi and Inertial Navigation Systems for Indoor Mobile Positioning," EURASIP Journal on Advances in Signal Processing, vol. 2006, pp. 1-12, 2006.

[23] [23] M. Hazas and A. Ward, "A Novel Broadband Ultrasonic Location System," pp. 264-280, 2002.

[24] [24] J. Sampedro, "SOCIEDAD Un GPS en el cerebro," pp. 4-5, 2013.

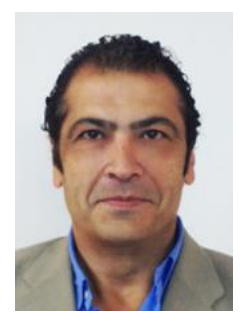

José Antonio Puertolás Montañés is MBA from IESE (University of Navarra), MS in Telecommunications Engineering (UPM) and MS in Technology Management from the University of Canterbury. He has thirty years of progressive leadership experience in telecommunications, IT on Internet, due to he enjoys an impressive record of achievement, with major recognized organizations worldwide due to he has spent the last 20 years creating and managing teams and high growth businesses worldwide. In June of 2012 he co-founded Mnopi to create a new value chain where the user is the owner of Internet. In turn, José is adviser of Veveo, Inc. and Innovation Advisory Council ZED group. Previously, he created Bizeu Consulting, to provide strategy, business development and sales to start-ups. He was also CEO and Invites cofounder of Networks. Its multimedia platform for mobile operators was recognized in 2005 by Guidewire Group as one of the top 20 innovative companies in Europe. In addition to co-founding Winphoria Networks. 


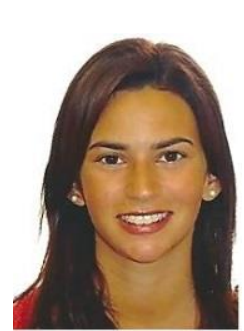

Adriana Mendoza Rodríguez has a degree in Geography by University of La Laguna, also she has a master's degree in Geographic Information Technologies by University Complutense of Madrid. Her project of final master consisted in the analysis of the area of influence of the stations of the Madrid metro. At present she is collaborator in the renewable energy division of the energetic, environmental and technological research centre, in which she participated in the drafting of articles about the optimization of solar energy in urban areas. Moreover she has attended several seminars that addressed issues of mobility and localization.

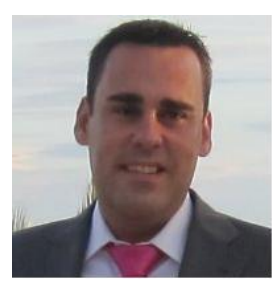

Iván Sanz Prieto is a member of Risk, Security and Compliance team (RSC) at AXA technologies. $\mathrm{He}$ received his degree in Computer engineering at Universidad Pontificia de Salamanca (UPSAM), his degree in Industrial engineering at the same university, and his $\mathrm{PhD}(\mathrm{ABD})$ in Web engineering and managing at Universidad International de La Rioja (UNIR). He has Master studies in IT Security at UPSAM and many IT Security certifications. Currently he is working in IT Security, dealing with ISO 27001, IT projects and supervising IT tasks. 